\title{
A study of packet scheduling algorithms in long term evolution-advanced
}

\author{
Mohd Mueen Ul Islam Mattoo, Huda Adibah Mohd Ramli \\ Department of Electrical and Computer Engineering, International Islamic University Malaysia (IIUM), \\ Malaysia
}

\begin{abstract}
Article Info
Article history:

Received Sep 21, 2018

Revised Mar 10, 2019

Accepted Jul 25, 2019

\section{Keywords:}

LTE-Advanced

Packet scheduling

Channel impairments

System capacity

CQI delay

ABSTRACT

The allocation of radio resources is one of the most critical functions performed by the Radio Resource Management (RRM) mechanisms in the downlink Long Term Evolution-Advanced (LTE-Advanced). Packet scheduling concerns itself with allocation of these radio resources in an intelligent manner such that system throughput/capacity can be maximized whilst the required multimedia Quality of Service (QoS) is met. Majority of the previous studies of packet scheduling algorithms for LTE-Advanced did not take the effect of channel impairments into account. However, in real world the channel impairments cannot be obliterated completely and have a direct impact on the packet scheduling performance. As such, this work studies the impact of channel impairments on packet scheduling performance in a practical downlink LTE-Advanced. The simulation results obtained demonstrate the efficacy of RM2 scheduling algorithm over other scheduling algorithms in maximizing the system capacity and is more robust on the effect of the cellular channel impairments.
\end{abstract}

Copyright () 2020 Institute of Advanced Engineering and Science. All rights reserved.

\section{Corresponding Author:}

Huda Adibah Mohd Ramli,

Department of Electrical and Computer Engineering,

International Islamic University Malaysia (IIUM),

Malaysia.

Email: hadibahmr@iium.edu.my

\section{INTRODUCTION}

As far as usage on a daily basis is concerned, the mobile phone far exceeds any other gadget that is presently being used by humans $[1,2]$. It is estimated that around $65 \%$ of the world population uses a mobile phone [3]. In the recent past, there has been a paradigm shift in the usage of the mobile phones from voice services to data-based services [4]. With the advent of portable computers, iPads, laptops with $3 \mathrm{G}$ modems and smart phones, the usage of wireless data is increasing with each passing day [5]. This in turn, demands for continuous advancements and innovations in technologies related to wireless communication in order to provide better Quality of Service $(\mathrm{QoS})$ and support more subscribers $[6,7]$.

Long Term Evolution-Advanced (LTE-Advanced) is one of the wireless communication systems developed to meet the stated demands [8]. However, the radio resources available in the LTE-Advanced is limited and it is a challenge for the LTE-Advanced operators to provide the best standard of services in terms of coverage, capacity, efficiency and QoS [9]. An efficient Radio Resource Management (RRM) function such as packet scheduling plays a vital role in maintaining these four standards in the LTE-Advanced system. Packet scheduling requires an algorithm to decide the packets that are to be transmitted to a particular user and distributes the radio resources efficiently such that the QoS of multimedia services are maintained while the throughput and capacity are maximized [10].

A number of packet scheduling algorithms have been developed [11-19]. For example, the classic Maximum-Largest Weighted Delay First (M-LWDF) [15] is one of the packet scheduling algorithms that 
showed an excellent performance in the legacy wireless communication systems. The M-LWDF selects a user to receive its packets based on its channel quality, average throughput and packet delay. However, the MLWDF as well as a majority of classic packet scheduling algorithms were developed for the wireless systems that assumed all transmitted packets are correctly received at the users. This is not a practical assumptions as the wireless systems are prone to various impairments and some transmitted packets may be erroneously received and require retransmission. Therefore a new packet scheduling algorithm which incorporated the need for retransmission of packets needs to be developed. It would be of interest to pin down a packet scheduling algorithm which (re)transmits packets in the most proficient manner and does justice between the old packets that need retransmission and new packets that require transmission for the first time while taking the channel impairments into consideration. Hence, the study evaluates and compares the classic packet scheduling algorithms for providing satisfactory QoS for more multimedia users in downlink LTE-Advanced. The downlink LTE-Advanced was chosen in this study due to a higher volume of multimedia services in that direction. The contribution of this study is to identify a robust packet scheduling algorithm that can maximize capacity while minimizing the detrimental effects of channel impairments towards the QoS of multimedia services.

The rest of the paper is structured in four sections. Section 2 discusses various packet scheduling algorithms used for LTE-Advanced. Section 3 explains the method used for this research followed by analysis of results in Section 4. The conclusion of the conducted work is given in Section 5.

\section{RELATED WORK}

Nowadays, the networks mostly need to deal with the multimedia applications. As such, it is important that the packet scheduling algorithms are aware of the QoS standards to be achieved. Additionally, in order to support the improved data rate offered by the LTE-Advanced there is a need for an efficient packet scheduling algorithm. The classic packet scheduling algorithms including the M-LWDF were developed for the TimeDivision Multiple Access (TDMA)-based wireless systems. In these TDMA-based systems, only one user is selected to receive its packets in each scheduling interval. This is in contrary to the downlink LTE-Advanced system that allows more than one users to receive its packets in each scheduling interval.

Therefore, a number of works modified the classic packet scheduling algorithms in order to support packet scheduling at each scheduling interval, at each frequency and on each Component Carrier (CC). It should be noted that the LTE-Advanced supports packet transmissions on multiple CCs or frequency bands and the minimum radio resource that is available for use by the competing users is known as a Resource Block (RB). An RB is of $180 \mathrm{kHz}$ bandwidth and extends up to $1 \mathrm{~ms}$ duration. These include the Channel Aware Queue Aware (CAQA) [17], Cross-layer Based Queue-Aware (CBQA) [18], Robust M-LWDF1 (RM1) and Robust M-LWDF2 (RM2) [19] which were the modified versions of the M-LWDF algorithm. These modified versions of M-LWDF scheduling algorithms are discussed next.

\subsection{Channel Aware Queue Aware (CAQA) [17]}

LTE-Advanced systems which cater to the high-speed multimedia services need an enhanced throughput performance in addition to maintaining the QoS requirement of the various applications. This compounds the issue of high data rate transmission, which usually comes with a low fairness and delay performances. CAQA is a scheduling algorithm that provides good throughput performance without a serious setback to the QoS requirement of the multimedia services. In other words, a good trade-off is achieved between throughput, fairness and QoS requirements.

The scheduling algorithm utilizes a cross layer interactions approach to make the scheduling decisions (i.e. the data rate from the physical layer, the QoS factor (delay deadline) from the application layer and Headof-Line (HOL) packet delay from the Medium Access Control (MAC) layer). The scheduling decisions are carried out in accordance with (1)

$$
k=\arg \max \left(r_{i, j, k}(t) \exp \left(\frac{T_{i}-W_{i}(t)}{2+W_{i}(t) * r_{i, j, k}(t) * T_{i}}\right)\right)
$$

where $r_{i, j, k}(t)$ represents the instantaneous data rate of the user $i$ on $\mathrm{CC} j$ on $\mathrm{RB} k$ at time $t, T_{i}$ represents the delay threshold of the user $i$ and $W_{i}(t)$ represents the HOL packet delay of user $i$ at time $t$.

The algorithm tends to maximize the instantaneous data rate enhancing the throughput performance of the system. Simultaneously, the algorithm makes use of the ratio of difference between delay threshold of the user and HOL packet delay to the product of the HOL packet delay, instantaneous data rate and delay threshold ensuring improved fairness and satisfactory QoS performance. 


\subsection{Cross-layer Based Queue-Aware (CBQA) [18]}

The increase in the throughput performance of any scheduling algorithm comes at the cost of low fairness and delay performance. The CBQA scheduling algorithm is designed in such a manner so that system throughput can be increased without making a compromise with the fairness and delay performance of the network. The CBQA algorithm makes the scheduling decisions according to the following mathematical equation.

$$
k=\arg \max \left(\frac{r_{i, j, k}(t)}{R_{i}(t)} \exp \left(\frac{T_{i}-W_{i}(t)}{W_{i}(t) * T_{i}}\right)\right)
$$

where $r_{i, j, k}(t)$ represents the instantaneous data rate of the user $i$ on $\mathrm{CC} j$ on $\mathrm{RB} k$ at time $t, R_{i}(t)$ represents the average throughput of user $i$ at time $t, T_{i}$ represents the delay threshold of the user $i$ and $W_{i}$ represents the HOL packet delay of user $i$ at time $t$.

As is evident from the above equation, the algorithm under discussion makes use of two ratios to make the scheduling decisions. Firstly, it uses the ratio of instantaneous data rate to average throughput, which ensures a good fairness and throughput performance. Secondly, the algorithm utilizes the ratio of difference between the delay deadline and HOL packet delay to the product of the two, ensuring an enhanced QoS performance. Thus, the algorithm provides a good trade-off point between the competing parameters.

\subsection{Robust M-LWDF1 (RM1) [19]}

The Robust MLWDF1 algorithm prefers the retransmission of packets over transmission of new packets. The RM1 algorithm operates in two parts. The first part of the algorithm always starts by determining if there are users available for retransmission. If yes, then priority of users for packets retransmission are determined randomly. It should be noted that only one user is allowed to receive its packets in each CC. Thereafter, the packets of the selected users are retransmitted. The second part of the algorithm comes into play only when retransmitting packets are absent. The scheduling decisions for the new packets of a user are made according to the following equations and all of the available RBs will be used for transmission of these new packets:

$$
\begin{aligned}
& k=\arg \max \left(a_{i} * W_{i}(t) \frac{a v g_{-} r_{i, j}(t)}{R_{i}(t)}\right) \\
& a v g_{-} r_{i, j}(t)=\frac{1}{R B_{\max }} * \sum_{k=1}^{k=R B \max } r_{i, j, k}(t)
\end{aligned}
$$

where $a_{i}$ represents the QoS requirement of user $i, W_{i}$ represents the HOL packet delay of user $i$ at time $t, r_{i, j, k}(t)$ represents the instantaneous data rate of the user $i$ on $\mathrm{CC} j$ on $\mathrm{RB} k$ at time $t, R_{i}(t)$ represents the average throughput of user $i$ at time $t$ and $R B_{\text {max }}$ represents the maximum number of RBs available.

The RM1 algorithm makes the scheduling decision for one $\mathrm{CC}$ at a time. It is worth mentioning that when a new user packet is transmitted, the second step of the algorithm stores the information about the Modulation and Coding (MCS) used. In case the packet is lost or received in error the same data will be utilized for retransmission of the packet.

\subsection{Robust M-LWDF2 (RM2) [19]}

Another modified version of M-LWDF algorithm is the RM2 algorithm. This algorithm gives equal opportunity to the new and retransmitting users. The working of this algorithm consists of four steps. Step 1 involves the determination of the nature of packets to be transmitted. If retransmitting packets are present, then the algorithm calculates the number of required Resource Blocks $\left(R B_{r e q}\right)$ needed to transmit them. If $R B_{r e q}$ is less than the RBs available in the $C C$, the user will be included in the active list. However, a new user is added to the active list by default. The second step involves the selection of that user according to Equation 3 . In the third step, if a retransmitting user has been selected, this step will randomly assign the $R B_{\text {req }}$ to that user. It has been observed that the random allocation of the RBs increases the system throughput. The final process involved in this step is to update the list of available RBs and the buffer.

Step 4 comes into play only when a new user has been chosen in second step. The allocation of RBs is performed in such a way so as to select the RB with best channel conditions, minimizing the number of RBs required for transmission. Thereafter, the RBs that are allocated are removed from the list of available RBs. It also involves updating the user buffer at the base station. The step is executed until all the new packets are 
transmitted or there are no more RBs available. Lastly, this step stores the information regarding the first transmission of packets which will be utilized in case the packets need retransmission. RM2 algorithm offers some additional advantages than RM1 scheduling algorithm where it can schedule more than one user on each $\mathrm{CC}$ in one scheduling interval.

\section{RESEARCH METHOD}

The computer simulation is an economical method of modelling a mobile cellular network, simple to implement and it allows full control to study the behavior of traffic flow when compared to other methods of modelling. For this research work, computer simulation method was chosen in light of the aforementioned reasons. This simulator makes a number of assumptions in order to minimize the complexity involved in modelling the downlink LTE-Advanced and due to time limitations. It is worth mentioning here that these assumptions are in line with the previous research works conducted in this domain. The simulation modelled a macro cell downlink LTE-Advanced of $0.5 \mathrm{~km}$ radius. The users are distributed uniformly in the cell and move in a fixed direction with a speed of $30 \mathrm{~km} / \mathrm{h}$. At the start of the data session the direction of motion for a user is set randomly and on reaching the boundary of the cell in that direction the users are wrapped around to make sure they stay in the area of simulation. The various other parameters for modelling the downlink LTEAdvanced are similar to [20-24].

This paper considered the Real Time (RT) video streaming application of $256 \mathrm{kbps}$ average data rate for study [25]. The video is one such multimedia application which of late has become one major partaker in consumption of LTE-Advanced radio resources. It is of interest to note that the minimum Average Throughput that needs to be maintained for video streaming application has been assumed to be $234.7 \mathrm{kbps}$. The value is selected on the basis that it is the minimum data rate that needs to be maintained in order to conduct a video session of two minutes for each user without its buffer running out of resources (in case upon initiation of the video session the size of de-jitter buffer is assumed full at $10 \mathrm{~s}$ duration). It has further been assumed in this simulation setup that the buffer delay threshold should not exceed $100 \mathrm{~ms}$.

\section{RESULTS AND ANALYSIS}

Packet scheduling algorithms play a vital role in maintaining a balance between QoS requirement and proficient utilization of the radio resources available to LTE-Advanced network. Additionally, given that the video application was chosen for study, the selected metrics must define the performance of RT applications in a wholesome manner. As such, this paper evaluated the performance of the packet scheduling algorithms on the basis of Packet Loss Ratio (PLR) and the Average Throughput. These metrics are considered common when evaluating the performance of the packet scheduling supporting the RT multimedia applications. Given that the CAQA and CBQA algorithms did not discuss how retransmission packets are handled, this study assumes that, packets of users that require retransmission are prioritized as compared to new packets, which is a similar method being implemented as in RM1 algorithm. However, contrary to RM1 algorithm that selects only a user for to receive retransmitted packets, the CAQA and CBQA algorithm allow more than a user to receive retransmitted packets in each scheduling interval an on each $\mathrm{CC}$.

The performance study is divided into two phases. At first, the performance of these algorithms for maximizing the system capacity without compromising the QoS of RT application is investigated. Thereafter, the detrimental effects of channel impairments on the QoS of RT applications is studied. In this phase, the most robust packet scheduling algorithm that can minimize these detrimental effects is pinned down.

\subsection{Performance of Packet Scheduling Algorithms with Increasing System Capacity}

Figure 1 illustrates the PLR performance of the packet scheduling algorithms with increasing system capacity. With the increase in the system capacity, more packets are being discarded by the schedulers, increasing the PLR. This can be attributed to the fact that when more users are available, more packets have to wait for (re)transmission at the buffer of the base station. As the radio resources available to the system are limited, it can be seen from Figure 1 that there is a sharp rise in the PLR beyond 50 users. This is because more packets waiting for (re)transmission approach the delay threshold and the schedulers discard these packets. It must be noted here that the PLR for RT multimedia services need to be below $10^{-3}$ so that the required QoS of RT services is satisfied. It can be seen from Table 1 that RM2 scheduling algorithm supports about $67.74 \%$ more users than the RM1 scheduling algorithm. The percentage improvements of the RM2 algorithm over CBQA and CAQA scheduling algorithms is $40.54 \%$ and $33.33 \%$, respectively for delay threshold of $10^{-3}$.

Figure 2 compares the capacity of the system with the Average Throughput of users. RM1 algorithm shows the worst performance among the four scheduling algorithms, while the other three show a similar performance. From Table 2 it can depicted that in terms of Average Throughput the RM2 algorithm 
outperforms the RM1 scheduling algorithm by a margin of $25.53 \%$. However, the margin of improvement over CBQA and CAQA algorithms are not that prominent. The percentage improvements being $5.36 \%$ and $3.51 \%$, respectively.

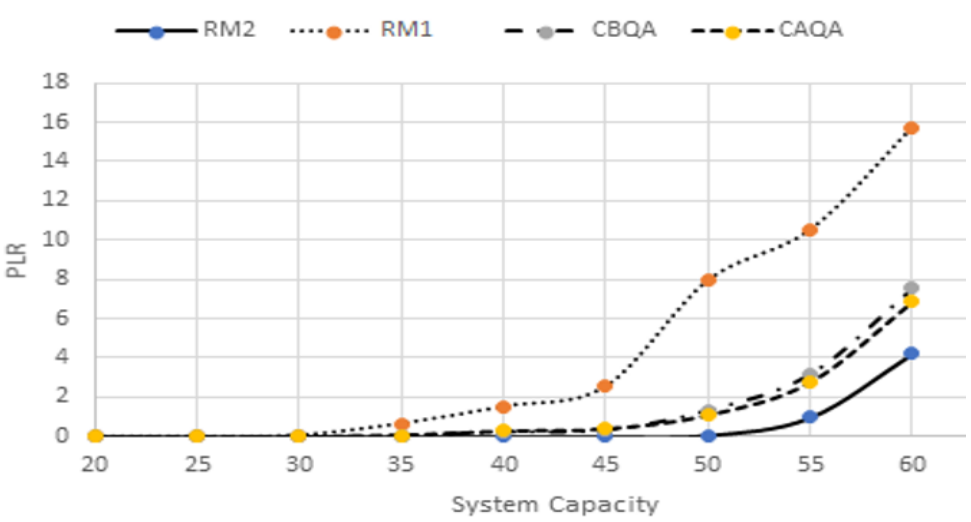

Figure 1. PLR vs System Capacity

Table 1. Maximum System Capacities/PLR threshold of 10-3

\begin{tabular}{ccc}
\hline $\begin{array}{c}\text { Packet Scheduling } \\
\text { Algorithm }\end{array}$ & $\begin{array}{c}\text { Maximum System } \\
\text { Capacity }\end{array}$ & $\begin{array}{c}\text { RM2 improvement over other } \\
\text { scheduling algorithms (\%) }\end{array}$ \\
\hline RM1 & 31 & 67.74 \\
CBQA & 37 & 40.54 \\
CAQA & 39 & 33.33 \\
RM2 & 52 & - \\
\hline
\end{tabular}

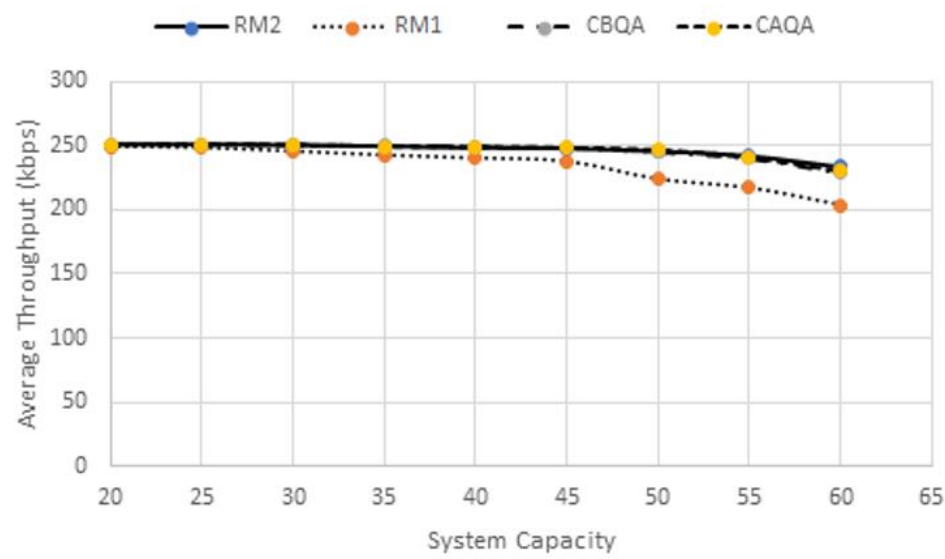

Figure 2. Average throughput vs System Capacity

Table 2. Maximum System Capacities to support Minimum Average Throughput of $234.7 \mathrm{kbps}$.

\begin{tabular}{ccc}
\hline $\begin{array}{c}\text { Packet Scheduling } \\
\text { Algorithm }\end{array}$ & $\begin{array}{c}\text { Maximum System } \\
\text { Capacity }\end{array}$ & $\begin{array}{c}\text { RM2 improvement over other } \\
\text { scheduling algorithms (\%) }\end{array}$ \\
\hline RM1 & 47 & 25.53 \\
CBQA & 56 & 5.36 \\
CAQA & 57 & 3.51 \\
RM2 & 59 & - \\
\hline
\end{tabular}




\subsection{The Impact of Channel Impairments on Packet Scheduling Performance}

It is assumed in this performance comparison that the channel is impaired due to the delay in Channel Quality Information (CQI) report. The CQI delay leads to packets been received in error as the CQI available at the base station may be different from the CQI that is reported due to channel variation as well as user mobility. It must be noted here that the number of users or system capacity is kept constant throughout this phase of study so as to make the comparison between competing algorithms more relevant.

Figure 3 draws a comparison between the competing algorithms based on varying CQI delay and PLR. The increase in PLR with the increasing CQI delay can be attributed to the increase in the delay between the CQI reported by the user and the actual transmission. The Modulation and Coding (MCS) selected for that user starts to become more and more irrelevant resulting in decadence in the faithful transmission of packets. Furthermore, it can be observed that RM2 scheduling algorithm can tolerate CQI delay up to $61 \mathrm{~ms}$ whereas RM1 can tolerate a CQI delay up to $3 \mathrm{~ms}$, CBQA for $12 \mathrm{~ms}$ and CAQA scheduling algorithm for $18 \mathrm{~ms}$. These statistics show the robustness of RM2 scheduling algorithm against CQI delay. It can be deduced from Table 3 that RM2 scheduling algorithm outperforms the other algorithms under study by overwhelming percentages of $1933.33 \%, 408.33 \%$ and $238.88 \%$ for RM1, CAQA and CBQA scheduling algorithms, respectively.

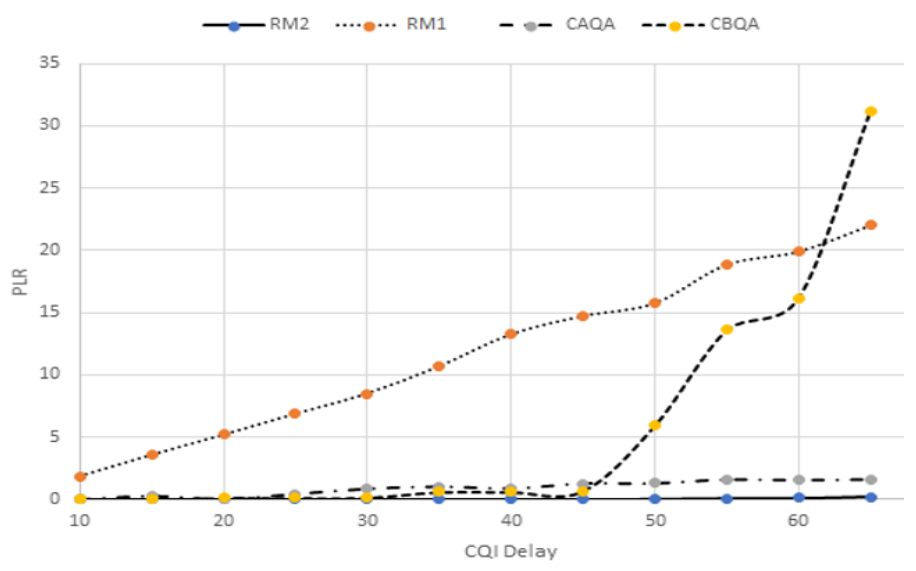

Figure 3. PLR vs. CQI Delay

Table 3. Maximum CQI Delay in ms for minimum PLR threshold of 10-3

\begin{tabular}{ccc}
\hline $\begin{array}{c}\text { Packet Scheduling } \\
\text { Algorithm }\end{array}$ & CQI Delay & $\begin{array}{c}\text { RM2 improvement over other } \\
\text { scheduling algorithms (\%) }\end{array}$ \\
\hline RM1 & 3 & 1933.33 \\
CAQA & 12 & 408.33 \\
CBQA & 18 & 238.88 \\
RM2 & 61 & - \\
\hline
\end{tabular}

Figure 4 illustrates the performance of the competing algorithms in terms of Average Throughput in presence of increasing CQI delay. The RM1 scheduling algorithm shows a progressive depreciation with an increasing CQI delay. The CAQA scheduling algorithm shows a much better performance than the other two and falls short of the RM2 scheduling algorithm only by a small margin. The performance of the CBQA scheduling algorithm is in line with the CAQA scheduler till a CQI delay of $45 \mathrm{~ms}$, after which it starts to deteriorate very quickly. It can be observed from Table 4 that the RM2 and CAQA scheduling algorithms outperform RM1 scheduling algorithm by a margin of more than $225 \%$. The improvement margin of RM2 and CAQA scheduling algorithm over CBQA scheduler is more than $30 \%$.

As is evident from the performance comparisons of the scheduling algorithms under study, it can be concluded that the RM2 scheduling algorithm outperforms the other scheduling algorithms in terms of all the evaluating parameters considered for this study. The performance improvement of RM2 scheduling algorithm over the RM1 scheduling algorithm can be understood from the fact that RM1 scheduling algorithm allocates all available RBs in a CC for (re) transmission to a selected user in which some of the RBs might not be utilized, which leads to wastage of already limited radio resources. This problem is further compounded when the same MCS is put to use for retransmission of those packets. The cross layer-based approaches considered in this

A study of packet scheduling algorithms in long term evolution-advanced (Mohd Mueen Ul Islam Mattoo) 
study i.e. CBQA and CAQA further the cause of the attaining a decent trade-off between the QoS requirement and the high data rate of the LTE-Advanced system. The two approaches provide the same transmission opportunity to retransmitting and new packets like that of RM2 scheduling algorithm. However, CBQA and CAQA scheduling algorithms involve an exponential function in their mathematical equations which causes the effects of delay to be more pronounced than the RM2 scheduling algorithm. This causes their performance to deteriorate when a certain delay threshold is crossed. The outperformance of the RM2 scheduling algorithm can be explained by the fact that it can schedule more than one user on each $\mathrm{CC}$ in one scheduling interval which leads to the efficient utilization of the radio resources. This seems to be the prime advantage of the winning algorithm over the other scheduling algorithms.

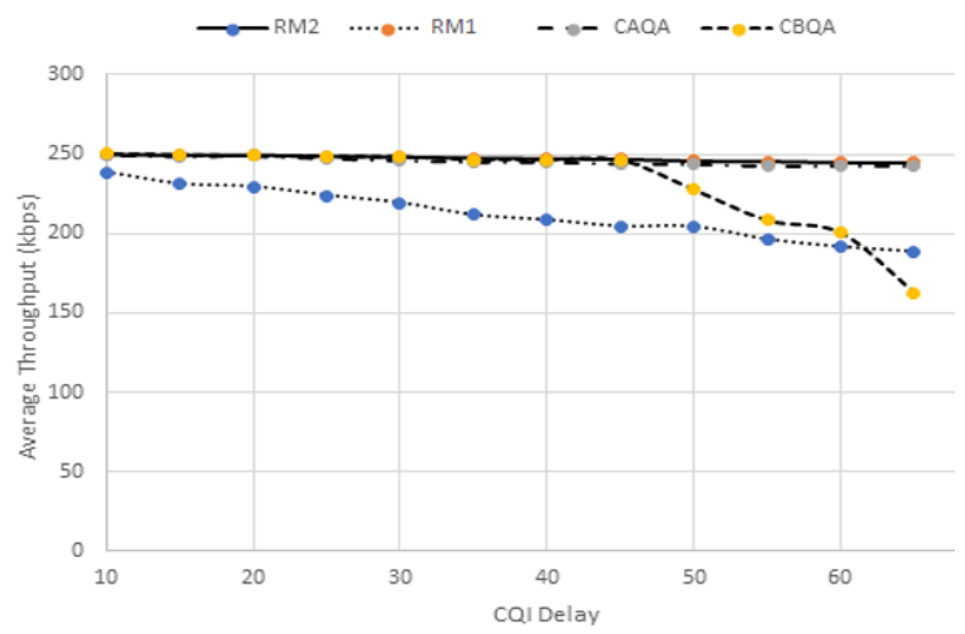

Figure 4. Average Throughput and CQI Delay

Table 4. Maximum CQI in ms for Minimum Average Throughput of $234.7 \mathrm{kbps}$.

\begin{tabular}{ccc}
\hline $\begin{array}{c}\text { Packet Scheduling } \\
\text { Algorithm }\end{array}$ & CQI Delay & $\begin{array}{c}\text { RM2 improvement over other } \\
\text { scheduling algorithms }(\%)\end{array}$ \\
\hline RM1 & 20 & $>225$ \\
CAQA & $>65$ & - \\
CBQA & 50 & $>30$ \\
RM2 & $>65$ & - \\
\hline
\end{tabular}

\section{CONCLUSION}

Packet scheduling forms a corner stone of RRM mechanisms as it is responsible for efficient allocation of radio resources among the users and hence needs sufficient attention from the research community. Additionally, the need to maintain a certain QoS for a LTE-Advanced application at an improved data rate calls for mechanisms that can schedule the packets more proficiently. The main contribution of this paper is to identify a classic packet scheduling algorithm that can maximize system capacity whilst providing satisfactory RT QoS and is robust to channel impairments. Simulation results suggest the efficacy of the RM 2 scheduling algorithm against the other scheduling algorithms considered for this research work. The RM2 scheduling algorithm outperforms the other scheduling algorithms both in terms of system capacity as well as robustness against the channel impairments. The paper pins down an optimum scheduling algorithm for a single cell scenario. The potential future research work may include the inclusion of multi-cell scenarios. The simulation conducted wraps the users into the same cell upon reaching the cell boundary. Since, handover is an important RRM function, therefore the performance of the packet scheduling algorithms can be studied while considering handover, which can greatly improve the system performance of downlink LTE-Advanced systems.

\section{ACKNOWLEDGEMENTS}

We are very grateful to Kementerian Pendidikan Malaysia for supporting this research under the grant ID of FRGS/1/2019/TK04/UIAM/02/2. 


\section{REFERENCES}

[1] S. F. S. Adnan, M. A. M. Isa, and H. Hashim, "Testbed versus simulation approach on RF communication with AA $\beta$ asymmetric encryption scheme on internet of things devices," Indones. J. Electr. Eng. Comput. Sci., vol. 14, no. 1, pp. 353-359, 2019.

[2] A. A. Z. Ibrahim and F. Hashim, "An architecture of 5G based on SDN NV wireless network," Indones. J. Electr. Eng. Comput. Sci., vol. 14, no. 2, pp. 725-734, 2019.

[3] S. Kemp, "Digital in 2018. Internet Users Pass the 4 Billion Mark," [Web log post] Retrieved from https://wearesocial.com, 2018.

[4] L. Chen, W. Chen, X. Zhang, and D. Yang, "Analysis and simulation for spectrum aggregation in LTE-advanced system," in IEEE Vehicular Technology Conference (VTC Fall), 2014, pp. 1-6.

[5] E. Dahlman, S. Parkvall, and J. Skold, 4G: LTE/LTE-advanced for mobile broadband. Academic press. Academic Press, 2013.

[6] A. Ghosh, R. Ratasuk, B. Mondal, N. Mangalvedhe, and T. Thomas, "LTE-advanced: next-generation wireless broadband technology," IEEE Wirel. Commun., vol. 17, no. 3, 2010.

[7] Asha and G. Mahadevan, "An Adaptive Cross-Layer Architercture to Optimize QoS Provisioning in MANET," Indones. J. Electr. Eng. Comput. Sci., vol. 6, no. 1, pp. 16-25, 2017.

[8] C.-C. Lin, K. Sandrasegaran, R. H.A.M., and M. Xue, "Requirement of handover modeling in the downlink 3GPP long term evolution system," in 4th International Conference on Advanced Information Networking and Applications Workshop, 2010, pp. 305-310.

[9] P. E. Mogensen et al., "LTE-Advanced: The path towards gigabit/s in wireless mobile communications," in Wireless Communication, Vehicular Technology, IEEE 1st International Conference on Information Theory and Aerospace \& Electronic Systems Technology, 2010, pp. 147-151.

[10] W. A. N. W. Abdullah, N. Yaakob, B. R. Ahmad, M. E. Elobaid, and S. A. Yah, "Corrupted packets discarding mechanism to alleviate congestion in wireless body area network," Indones. J. Electr. Eng. Comput. Sci., vol. 14, no. 2, pp. 581-587, 2019.

[11] B. S. Tsybakov, "File Transmission over Wireless Fast Fading Downlink," IEEE Trans. Inf. Theory, vol. 48, no. 8, pp. 2323-2337, 2002.

[12] A. Akhtar and H. Arslan, "Downlink resource allocation and packet scheduling in multi-numerology wireless systems," 2018 IEEE Wirel. Commun. Netw. Conf. Work. WCNCW 2018, pp. 362-367, 2018.

[13] et al. Dahlman, E., 3G Evolution: HSPA and LTE for Mobile Broadband. Elsevier Ltd., 2007.

[14] et al. Jalali, A., "Data Throughput of CDMA-HDR a High Efficiency-High Data Rate Personal Communication Wireless System," in IEEE 51 st Vehicular Technology Conference Proceedings, 2000, pp. 1854-1858.

[15] A. Mathew, K. Kumaran, K. Ramanan, A. Stolyar, and P. Whiting, "Providing Quality of Service over a Shared Wireless Link," IEEE Commun., vol. 39, no. 2, pp. 150-154, 2001.

[16] G. Barriac and J. Holtzman, "Introducing Delay Sensitivity into the Proportional Fair algorithm for CDMA Downlink Scheduling," in IEEE Seventh International Symposium on Spread Spectrum Techniques and Applications, 2002, pp. 652-656.

[17] G. Aiyetoro and F. Takawira, "A Cross-layer based packet scheduling scheme for multimedia traffic in satellite LTE networks," in IEEE 6th International Conference on New Technologies, Mobility and Security (NTMS), 2014, pp. 16.

[18] G. Aiyetoro and F. Takawira, "A new user scheduling scheme in LTE/LTE-A networks using cross-layer design approach," in IEEE Military Communications Conference MILCOM, 2015, pp. 1-6.

[19] H. A. M. Ramli and F. N. M. Isa, "Improving real-time multimedia scheduling in practical mobile cellular channels," in IEEE 6th International Conference on Intelligent and Advanced Systems (ICIAS), 2016, pp. 1-6.

[20] M. I. H. Mansor, H. A. M. Ramli, A. L. Asnawi, and F. N. M. Isa, "Simulation of packet scheduling in cognitive long term evolution-advanced," Indones. J. Electr. Eng. Comput. Sci., vol. 8, no. 2, pp. 533-540, 2017.

[21] H. A. M. Ramli, A. L. Asnawi, F. N. M. Isa, A. W. Azman, and M. I. H. Mansor, "Investigations of component carrier selection algorithms in Long Term Evolution-Advanced," Indones. J. Electr. Eng. Comput. Sci., vol. 10, no. 1, pp. 330-336, 2018.

[22] H. A. M. Ramli, F. N. M. Isa, A. L. Asnawi, A. Z. Jusoh, and A. W. Azman, "Urgency-Aware Scheduling Algorithm for Downlink Cognitive Long Term Evolution-Advanced," in 2019 IEEE 89th Vehicular Technology Conference (VTC Spring), 2019, no. 1-5.

[23] H. A. M. Ramli and Z. I. Rizman, "Novel scheduling algorithm for optimizing real-time multimedia performance in Long Term Evolution-Advanced," Turkish J. Electr. Eng. Comput. Sci., vol. 25, no. 1, pp. 247-273, 2017.

[24] H. A. M. Ramli, K. Sandrasegaran, A. F. Ismail, S. . Latif, and F. N. M. Isa, "A Simulation Tool for Downlink Long Term Evolution-Advanced,” Res. J. Appl. Sci. Eng. Technol., pp. 2032-2041, 2014.

[25] K. Sandrasegaran, H. A. M. Ramli, and R. Basukala, "Delay-Prioritized Scheduling (DPS) for Real Time Traffic in 3GPP LTE System," in IEEE Wireless Communication and Networking Conference, 2010, pp. 1-5. 


\section{BIOGRAPHIES OF AUTHORS}
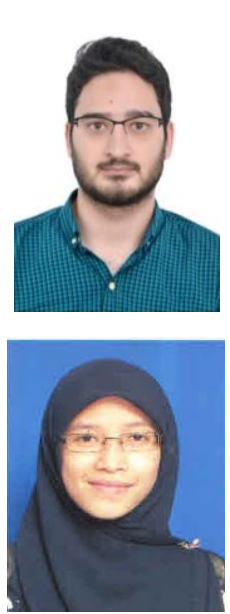

MOHD MUEEN UL ISLAM MATTOO finished his Masters degree in Communication Engineering at the International Islamic University of Malaysia in 2018. Previously, he graduated cum laude from the University of Kashmir in Electronics and Communication Engineering in the year 2014. Before embarking the field of research and academics, he worked as an apprentice and a field maintenance engineer in the subject field of wireless communication. Making his industry experience a platform for his academic career, he started his research in the same knowledge domain. His current research interests include: packet scheduling in LTE-advanced systems, carrier aggregation and cognitive radio networks.

HUDA ADIBAH MOHD RAMLI received the M.Sc. degree in Software Engineering from University of Technology Malaysia (Malaysia) in 2006, and the Ph.D. degree from University of Technology, Sydney (Australia) in 2012. Her research interests are mainly related to packet scheduling, resource management, radio propagation, carrier aggregation and cognitive radio in mobile cellular networks. 